\title{
Irritable Bowel Syndrome: A "Mesh" of a Situation
}

\author{
Matthew G. Barnes, MD
}

The diagnosis of irritable bowel syndrome (IBS) frequently is made after the exclusion of a mechanical etiology for a patient's symptoms. This case demonstrates that IBS symptoms can be caused by a rare complication of a common surgery: mesh herniorrhaphy repair. The patient is a 50-year-old woman who underwent periumbilical Marlex mesh herniorrhaphy 13 years before presentation. After her operation, the patient developed constipation (approximately one bowel movement per week) alternating with diarrhea for approximately 10 years. An abdominal radiograph showed large amounts of stool, and after a normal colonoscopy the patient was diagnosed with IBS. The patient was treated with tegaserod (Zelnorm) and polyethylene glycol (MiraLAX), which did not palliate her symptoms. The patient presented with obstructive symptoms and physical findings of an incarcerated umbilical hernia. A computed tomography (CT) scan of the abdomen confirmed an umbilical hernia involving a segment of small bowel with surrounding fecalization of enteric contents. During operative repair, the patient was found to have Marlex mesh fully eroded into the lumen of the small bowel, causing a partial obstruction. The involved section of small bowel was resected, and during serial follow-up the patient had complete resolution of her IBS-like symptoms. A discussion follows regarding the implications of mesh migration, and questions are posed for future research. (J Am Board Fam Med 2012;25:120-123.)

Keywords: Case Report, Hernioplasty, Herniorrhaphy, Irritable Bowel Syndrome, Polypropylene, Surgical Mesh

\section{Case History}

$\mathrm{KT}$ is a 50 -year-old woman with a 10 -year history of irritable bowel syndrome (IBS) symptoms (alternating diarrhea and constipation); she had a periumbilical herniorrhaphy 13 years earlier. KT presented to the general surgery service for an incarcerated periumbilical hernia. The patient presented with periumbilical pain that she rated 10 out of 10. She had associated nausea and vomiting for the past few days, and she specifically noted a recurrence of her hernia. Her periumbilical area was normal color but tender to the touch, and there was a protruding, nonreducible hernia.

KT's medical history is remarkable for diagnosed IBS, controlled hypertension, controlled esophageal reflux, and an incidental hiatal hernia.

This article was externally peer reviewed.

Submitted 23 August 2010; revised 24 April 2011; accepted 9 May 2011.

From the Medical Corps, United States Air Force, Dewitt Army Hospital, Fort Belvoir, VA.

Funding: none.

Conflict of interest: none declared.

Corresponding author: Matthew Barnes, Dewitt Army Hospital, Fort Belvoir, VA.
Her surgical history was remarkable for several abdominal surgeries: a Nissen fundoplication 10 years prior, a vaginal hysterectomy 20 years prior, an oophorectomy 16 years prior, an appendectomy approximately 30 years prior, and bladder suspension and revision 27 years and 24 years prior, respectively. Most notably, 13 years before her presentation, the patient had an elective periumbilical herniorrhaphy with placement of a nonanchored Marlex mesh plug (Marlex Pharmaceuticals, Inc., New Castle, DE) for a nonstrangulated periumbilical hernia. There were no perioperative or postoperative complications noted in the operative report or the postoperative notes.

Three years after the operation, the patient developed IBS symptoms: alternating constipation (having approximately 1 bowel movement per week) and diarrhea in association with feelings of bloating. The patient had an initial evaluation for IBS at the onset of her symptoms, which included an abdominal radiograph. Radiologist interpretation of the radiograph noted large amounts of stool without obstruction. This finding led to further investigation with colonoscopy, which was negative for any pathology. The patient then was diagnosed 
with IBS. KT was treated with tegaserod (Zelnorm, Novartis International AG, Basil, Switzerland) and polyethylene glycol (Miralax) for 10 years, but these drugs never palliated her symptoms. She continued this ineffective course until her presentation to the emergency room.

The patient had no remarkable drug allergies. She denied alcohol and tobacco use. Her family history was unremarkable. During examination, the patient was in pain. Her abdominal examination demonstrated a soft, nonreducible, tender incisional hernia. No skin erythema or duskiness was noted over the hernia site. The patient's abdomen was mildly distended, but she did not have any peritoneal signs.

A complete blood count and comprehensive metabolic panel were unremarkable, save for a mildly elevated white blood cell count of 11,000 . There was no anemia. A computed tomography scan of the abdomen demonstrated a periumbilical hernia with possible incarceration and a possible small bowel obstruction based on fecalization of enteric contents (see Figure 1). After a discussion of the risks and benefits of the procedure, KT was taken to the operating room for surgical repair of an entrapped periumbilical hernia.

During operative repair, the patient was found to have Marlex mesh fully eroded into the lumen of the small bowel, causing a partial obstruction: a mesh flap that would cause transient blockage and release of stool. The mesh also was adherent to the anterior abdominal wall at the region of the umbi-

Figure 1. Computed tomography scan of patient's abdomen with oral contrast. Arrow indicates area of fecalization, a sign of obstruction.

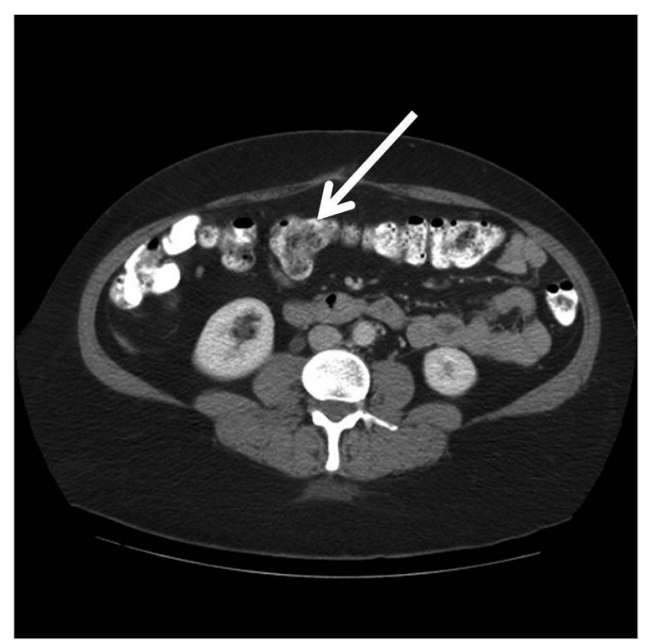

licus, with no other loops of bowel adhered to it. The involved section of small bowel was resected, and the recurrent hernia site was sutured closed.

Two days postoperatively, the patient noted she was having more frequent, formed bowel movements (approximately once a day). Her distension and feelings of bloating resolved. The patient even stated, "the operation cured my IBS." During a telephone conversation 6 months postoperatively, the patient stated that her IBS symptoms still were resolved. The surgical team made the conclusion that KT's IBS-like symptoms were caused by the Marlex mesh causing a transient physical obstruction, leading to the bloating, cramping, and the alternating diarrhea and constipation.

\section{Literature Search}

A PubMed search was performed using the term Marlex mesh migration, which returned 36 articles. These were limited to the English language articles that were appropriate for the topic (as noted by title and abstract). The citations of these articles were then searched and pulled in the same manner. A total of 14 appropriate articles were found and referenced.

\section{Discussion}

KT's symptoms were attributed to IBS for 10 years, when the etiology was likely migrating mesh, as suggested by her complete surgical "cure." At the onset of her symptoms, the patient did not meet any Manning criteria and her Rome criteria was only positive for changes in frequency of bowel movements. ${ }^{1,2}$ Because the abdominal radiograph and colonoscopy were inconclusive, the initial diagnosis of IBS was made by exclusion. In light of the patient's persistent symptoms refractory to medical therapy, further evaluation could have been pursued. The American Gastroenterological Association guidelines recommend evaluating after treatment failure by blood count, C-reactive protein, tissue transglutaminase, or a colonic transit study. ${ }^{3}$ In this case, a colonic transit study may have demonstrated the partial obstruction caused by the mesh. Other studies that are not suggested by the American Gastroenterological Association guidelines, such as a CT scan (by demonstrating obstruction), capsule endoscopy, or a small-bowel follow-through on colonoscopy, might also have demonstrated the migrating mesh. 
A review of medical literature demonstrates several case reports of Marlex mesh migration. Most frequently noted was that Marlex mesh can erode into the lumen of the bowel and cause acute obstructive symptoms, ${ }^{4-9}$ perforation, ${ }^{10}$ and volvulus. ${ }^{11}$ However, this is the first case report in which chronic IBS-like symptoms were a result of Marlex mesh obstruction, proven by resolution after resection.

Marlex mesh also can migrate into other structures: bladder, ${ }^{12}$ scrotum, ${ }^{13}$ and vas deferens. ${ }^{14}$ One study ${ }^{15}$ evaluated young men with azoospermia after inguinal herniorrhaphy and noted 14 cases of infertility caused by migrating mesh. Mesh migration often can mimic ovarian and colonic malignancy ${ }^{16,17}$ by creating a tissue "masses" noted on CT scan. The numerous accounts of mesh migration led Hamouda et $\mathrm{al}^{12}$ to conclude that this was a time-dependent and insidious effect, and that we were seeing "the tip of the iceberg" because complications from mesh migration may increase in prevalence over time.

Though there are mounting numbers of cases of mesh migration, it is assumed that many cases are missed. The overall rate of migration is unknown beyond that of case reports because most hernia mesh migration is insidious and difficult to detect with conventional imaging. The clinical significance can vary from absolutely no symptoms to silent symptoms (such as aforementioned azoospermia) to life-threatening conditions (like obstruction or perforation).

Though migration still is considered a complication of mesh herniorrhaphy (a risk that many surgeons counsel patients about preoperatively), many primary care providers are unaware of the possibility of mesh migration and may misdiagnose conditions caused by migration as different acute or chronic conditions. A provider should have high clinical suspicion for mesh migration if a previous hernia recurs. Likewise, in cases refractory to medical management, consider extended imaging, such as CT scan or colonic motility studies, which may lead to further work-up.

\section{Conclusion}

Have a high clinical suspicion for other causes of IBS-like symptoms if medication is ineffective. A meticulous history and physical examination should be pursued in every patient with potential IBS (or any chronic condition) to rule out a mechanical or otherwise treatable cause. In those with concerning histories, such as those with prior mesh herniorrhaphy, consider further evaluation per AGA guidelines or with further imaging. After all, IBSlike symptoms can occur for many different reasons, including reasons yet unknown.

Thank you to the editors of the Fournal of the American Board of Family Medicine for considering my work in progress. Thank you Drs. Joshua Hodge and Stephen Titus for mentoring me in this project. Thank you to Dr. Albaneze, Dr. Tyson Becker, and Dr. Mark Brisson for allowing me to work with you during my surgical rotation. Thank you to Dr. Marc Childress and Dr. Robert Marshall for your review of this poster. Finally, thank you to Dr. Brian Reamy, Dr. Mark Stephens, and Dr. Brian Unwin for your support.

\section{References}

1. Manning AP, Thompson WG, Heaton KW, Morris AF. Towards a positive diagnosis of the irritable bowel. Br Med J 1978;2:653-4.

2. Longstreth GF, Thompson WG, Chey WD, Houghton LA, Mearin F, Spiller RC. Functional bowel disorders. Gastroenterology 2006;130(5):1480-91.

3. American Gastroenterological Association. American Gastroenterological Association medical position statement: irritable bowel syndrome. Gastroenterology 2002;123:2105-7.

4. Lo DJ, Bilimoria KY, Pugh CM. Bowel complications after prolene hernia system repair: a case report and review of the literature. Hernia 2008;12:437-40.

5. Leblanc KA. Complications associated with the plug-and-patch method of inguinal herniorrhaphy. Hernia 2001;5:135-8.

6. Moorman M, Price P. Migrating mesh plug: complication of a well-established hernia repair technique. Am Surg 2004;70:298-9.

7. Muria A, Formisano V, Di Carlo F, Aveta A, Giglio D. Small bowel obstruction by mesh migration after umbilical hernia repair. Ann Ital Chir 2007;78: $59-60$.

8. Chuback JA, Singh RS, Sills C, Dick LS. Small bowel obstruction resulting from mesh plug migration after open inguinal hernia repair. Surgery 1999; 127:475-6.

9. Ferrone R, Scarone P, Natalini G. Late complication of open inguinal hernia repair: small bowel obstruction caused by intraperitoneal mesh migration. Hernia $2003 ; 7: 161-2$.

10. Benedetti M, Albertario S, Niebel T, et al. Intestinal perforation as a long-term complication of plug and mesh inguinal hernioplasty: case report. Hernia 2005;9:93-5.

11. Stout CL, Foret A, Christie B, Mullis E. Small bowel volvulus caused by migrating mesh plug. Am Surg 2007;73:796-7. 
12. Hamouda A, Kennedy J, Grant N, Nigam A, Karanjia N. Mesh erosion into the urinary bladder following laparoscopic inguinal hernia repair; is this the tip of the iceberg? Hernia 2010;14:317-9.

13. Chowbey P, Bagchi N, Goel A. Mesh migration into the bladder after TEP repair: a rare case report. Surg Laparosc Endosc Percutan Tech 2006;16:52-3.

14. Dieter R. Mesh plug migration into scrotum: a new complication of hernia repair. Int Surg 1999;84: 57-9.
15. Shin D, Lipshultz L, Goldstein M, et al. Herniorrhaphy with polypropylene mesh causing inguinal vasal obstruction. Ann Surg 2005;241:553-8.

16. Rettenmeier MA, Heinemann S, Truong H, Micha JP, Brown JV 3rd, Goldstein BH. Marlex mesh mimicking an adnexal malignancy. Hernia 2009;13: 221-3.

17. Ojo P, Abenthroth A, Fielder P, Yavorek G. Migrating mesh mimicking colonic malignancy. Am Surg 2006;72(12):1210-1. 